\title{
Enhancing the Performance of P3HT/Cdse Solar Cells by Optimal Designing of Active Layer
}

\author{
Fatemeh Rezaei ${ }^{1}$, Alireza Kashaniniya ${ }^{1}$, Reza Sabbaghi-Nadooshan ${ }^{1}$ \\ ${ }^{I}$ (Department of Electrical Engineering, Central Tehran Branch, Islamic Azad University, Tehran, Iran)
}

\begin{abstract}
The present study examined the influence of different condition like as doping, in active layer, on the performance of P3HT/CdSe Solar cells.In this work, we analyzed the best doping for the configuration of P3HT/ CdSe in order to improve the performance of the solar cell. For this aim, we investigated the current density of electrons, the electric field, the short-circuit current and the open-circuit voltage in different doping . The results indicate that when the doping is increased in P3Ht and is decreased in CdSe, the current density of electrons, the electric field, the short-circuit current, and the open-circuit voltage are increased. Finally, we obtained doping of $13 \times 10^{14} \mathrm{~cm}^{-3}$ and $16 \times 10^{14} \mathrm{~cm}^{-3}$ for electron and hole donor respectively as the best doping for this configuration.
\end{abstract}

Keywords: doping - electric field - efficiency - solar cells

\section{Introduction}

The rise of organic materials with semiconductive properties has encouraged the development of organic electronics based on novel electronic devices such as organic light-emitting diodes (OLED), organic solar cells (OSC), organic thin film transistors (OTFT) and etc. Many organic semiconductors can be processed in solutions and deposited layers easily by spinning, casting, printing and etc. This is one of the great advantages of organic semiconductors, because it reduces considerably the cost of electronic devices processing. On the other hand, organic semiconductors have very low charge carrier mobility because of their amorphous or semicrystalline structure. Thus, in organic solar cells the diffusion length of photogenerated carriers is very short because of the poor mobility and therefore, the conventional p-n junction has very limited efficiency for photovoltaic conversion. This drawback of organic semiconductors has been overcome with different solar cell configurations in which the p-n interface has an increased area. The typical configuration consists of a blend of a p-type organic semiconductor as P3HT (poly(3- hexylthiophene)) and an n-type one as PCBM (Phenyl-C61butyric acid methyl ester). In a proper blend, the interface area is maximized and each semiconductor must be connected to the respective contact to provide the electrical path to the photo-generated electrons and holes, after they are separated at the p-n interface. Another approach is to combine with inorganic semiconductors in organic-inorganic hybrid solar cells. For this, the great availability of inorganic semiconductor nanostructures allows the design of different hybrid solar cell configurations. One of the simplest configurations is the system of semiconductor nano particles with n-type conductivity, such as $\mathrm{CdS}$ and $\mathrm{CdSe}$, embedded in a polymeric matrix with p-type conductivity, such as P3HT .

In this paper, cadmium selenide is used as electron acceptor and $\mathrm{P} 3 \mathrm{Ht}$ as electron donor. First, the architecture of layers' structure is determined. Then for the purpose of validation, the paper is simulated using Silvaco, and its current-voltage curve is illustrated. Then the main idea of the paper, which is the change in the amount of doping in donor and acceptor, is presented and the voltage-current curves and quantum efficiency and cell efficiency are illustrated. Finally, the results and conclusion regarding the increase of efficiency, the increase of Voc and Isc are presented in the form of comparison tables.

\section{Model}

We utilized Bimolecular Langevin Recombination Model in order to determine recombination rate. [1] [2] [3] [4] [5] [6] The Langevin recombination rate coefficient has been given in literature by equation:

$r L \mathrm{x}, \mathrm{y}, \mathrm{t}=\mathrm{q} \varepsilon \varepsilon 0 u_{n} E+u_{p} E$

Where $u_{n}$ and $u_{p}$ are electron and hole mobility respectively. The electron-to-hole mobility ratio $\beta$ is defined by:

$$
\beta=u_{n} u_{p}
$$

The Langevin recombination rate is given by: 
$R L n, \mathrm{x}, \mathrm{y}, \mathrm{t}=r L \mathrm{x}, \mathrm{y}, \mathrm{t} n_{p}-n i 2$

Where $\mathrm{n}$ is electron density, $\mathrm{p}$ is hole density and $n_{i}$ is the intrinsic concentration .The Langevin recombination rate is included in recombination terms in the carrier continuity equations (equations 4 and 5). The continuity equations for electrons and holes are defined by equations:

$$
\begin{array}{r}
\partial \mathrm{n} \partial \mathrm{t}=1 q \operatorname{div} j_{n}+G_{n}-R_{n} \\
\partial \mathrm{p} \partial \mathrm{t}=1 q \operatorname{div} j_{p}+G_{p}-R_{p}
\end{array}
$$

Where $\mathrm{n}$ and $\mathrm{p}$ are the electron and hole concentration, $j_{n}$ and $j_{p}$ are the electron and hole current densities, $G_{n}$ and $G_{p}$ are the generation rates for electrons and holes, $R_{n}$ and $R_{p}$

the recombination rates for electrons and holes, and $\mathrm{q}$ is the magnitude of the charge on an electron. Furthermore, we used singlet and triplet model for exchange between charged carrier and singlet and triplet excitons (in SILVACO Atlas). Almost, ray tracing based on geometrical optic principles has been utilized in order to simulate optoelectronic devices. But in this case, we used Beam Propagation Method because we need a method that takes into account the wave nature of light. The BPM in (LUMINOUS in silvaco environment ) has been extended to solve a more general Helmholtz Wave Equation (Equation 6).

$\nabla 2 \mathrm{E}(\mathrm{r}, \mathrm{t})-\mathrm{n} 2 \mathrm{c} 2 \partial 2 E r, t \partial \mathrm{t} 2=0$

Here, $\mathrm{E}$ is the electric field of an optical wave, $\mathrm{n}$ is the complex refractive index of the material, and $\mathrm{c}$ is the speed of light in vacuum. We need to know almost all physical parameters of each layer in the organic solar cell in order to simulate parameters such as hole and electron mobility, band gap energy, density of state, LUMO, HOMO and dielectric constant.

We seek to simulate the polymer cell Zno/P3HT:CdSe/Mo in the two-dimensional silvaco atlas environment. Since polymer materials are limited in this software environment, we need to define the $\mathrm{P} 3 \mathrm{Ht}$ polymer material. one of the reasons for using $\mathrm{P} 3 \mathrm{Ht}$ is the small size of the band gap and its high donor ability compared with other polymers. We have also used Molybdenum because of its low thermal expansion coefficient, which will help the matter have a lower expansion when exposed to increased temperature. Also Zno with its high work function is considered a positive factor for increasing the efficiency.

In this work, we investigate the influence of material dopings on performance of proposed solar cells.

\section{Simulation Using Silvaco}

We are going to simulate the polymer cell Zno/P3HT:CdSe/Molybdenum in two-dimensional Silavo ATLAS simulator. Since polymer materials are limited in this software, the polymer P3Ht should be defined. One of the reasons for using P3Ht is the short length of the gap band and that it is a greater donor than other polymers. Molybdenum is also used for its low thermal expansion coefficient. This decreases the expansion of Molybdenum caused by the increase of temperature. Zno also has a high work function, which is considered a positive factor for the increase of efficiency. For cell simulation, we used singlet, langevin, pfmob, and print models for cell formation and simulation in Silvaco.

\section{The Effect of Doping In Electron Donor (P3HT) Layer}

Regulating the doping in electron donor is one effective way to improve the performance of our proposed cell . In order to analyzing this parameter, in begging we consider $10^{13} \mathrm{~cm}^{-3}$ for doping in electron donor.
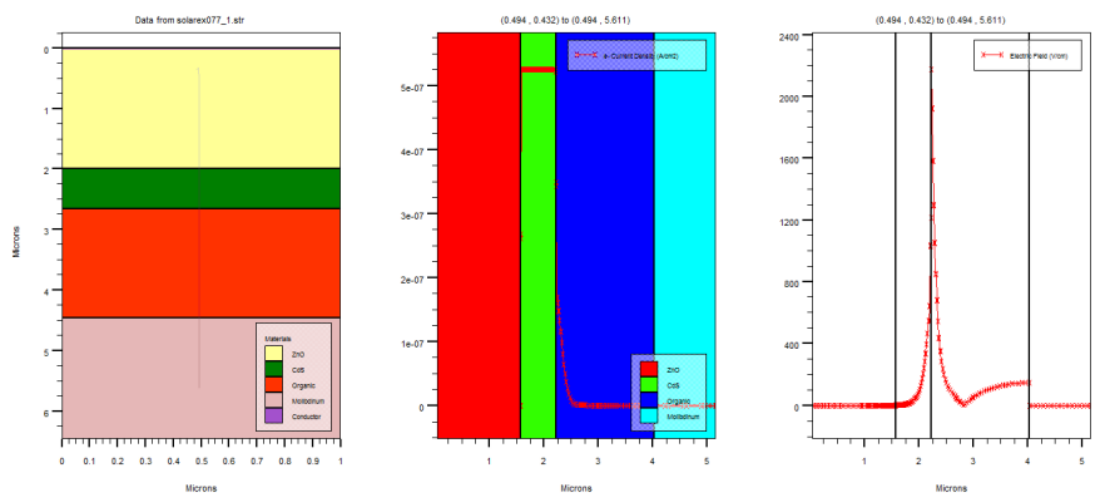

Fig1. Configuration, current density and electric field of the P3HT/CdSe solar cells with $10^{13} \mathrm{~cm}^{-3}$ for doping in electron donor. 
In next step, we consider $10^{16} \mathrm{~cm}^{-3}$ for doping in electron donor.
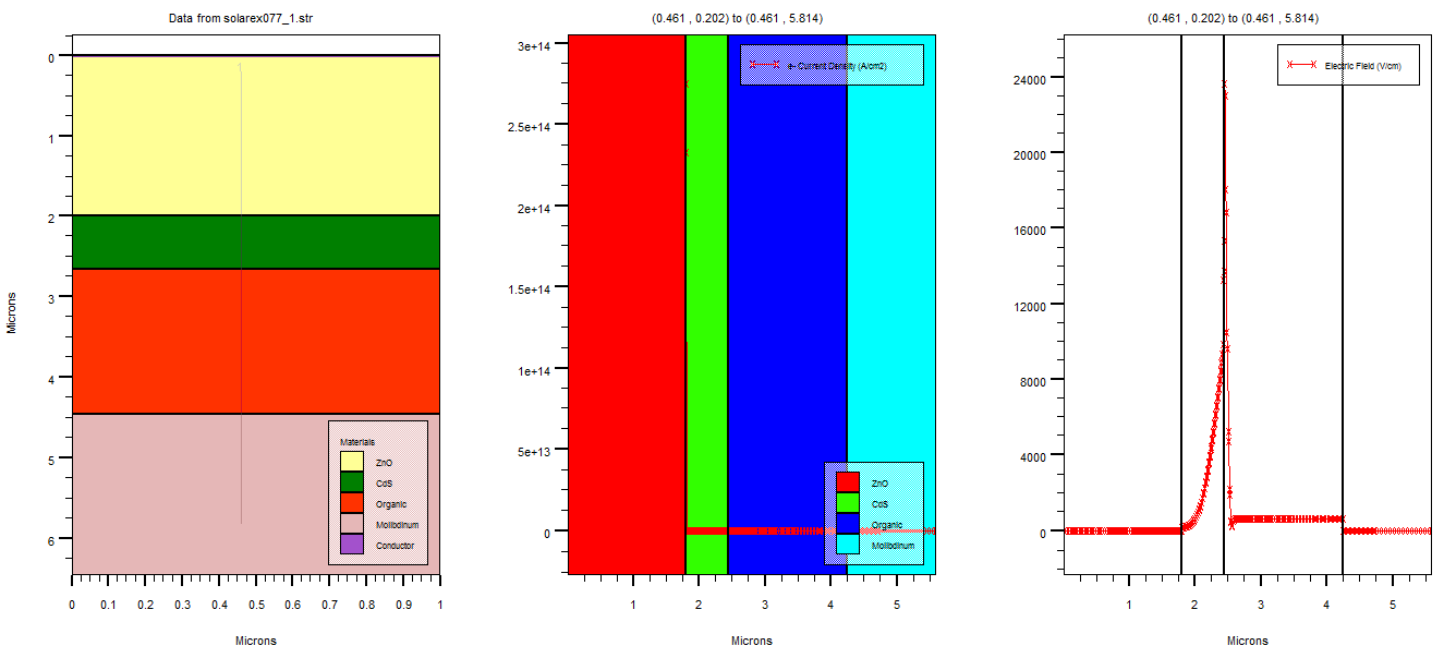

Microns

Microns

Fig2. Configuration, current density and electric field of the P3HT/CdSe solar cells with $10^{16} \mathrm{~cm}^{-3}$ for doping in electron donor.

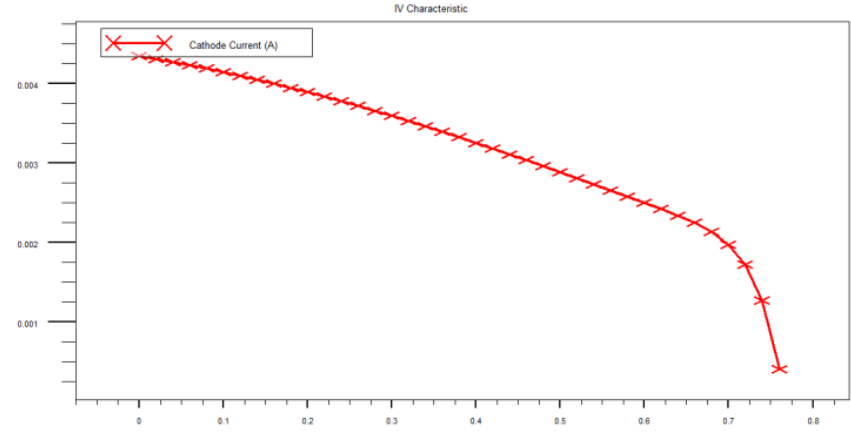

Fig3. Current- voltage curve of the $\mathrm{P} 3 \mathrm{HT} / \mathrm{CdSe}$ solar cells with doping $10^{16} \mathrm{~cm}^{-3}$ in electron donor.

Therefore, we should specify a optimize mount for doping in electron donor. There are different approaches for this aim such as neural network, Statistical calculations and trial and error, using equation.

We can see, specify a optimize mount for doping $13 \times 10^{14} \mathrm{~cm}^{-3}$ and $16 \times 10^{14} \mathrm{~cm}^{-3}$ in electron donor and hole donor, respectively.

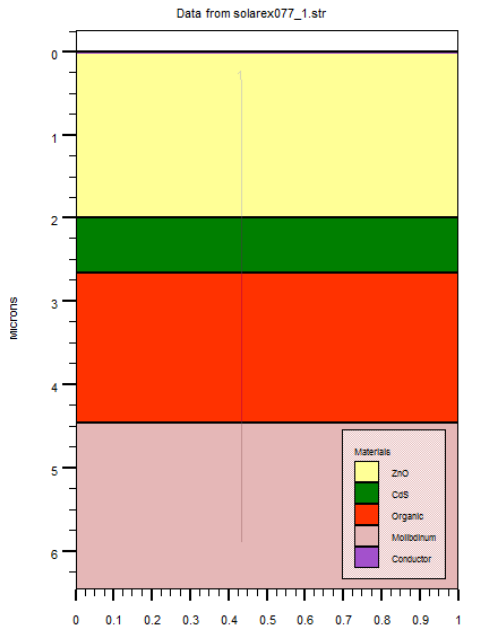

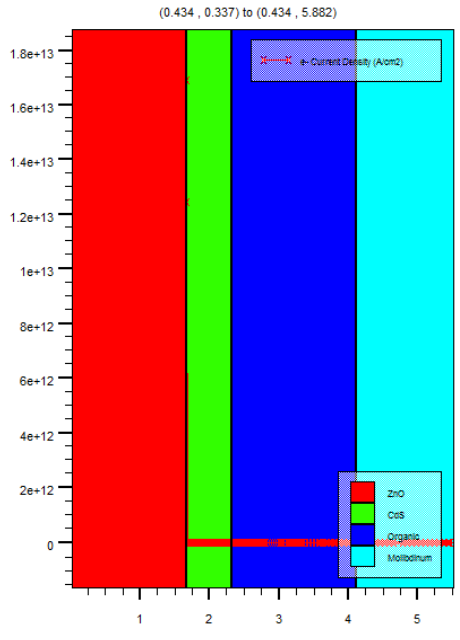

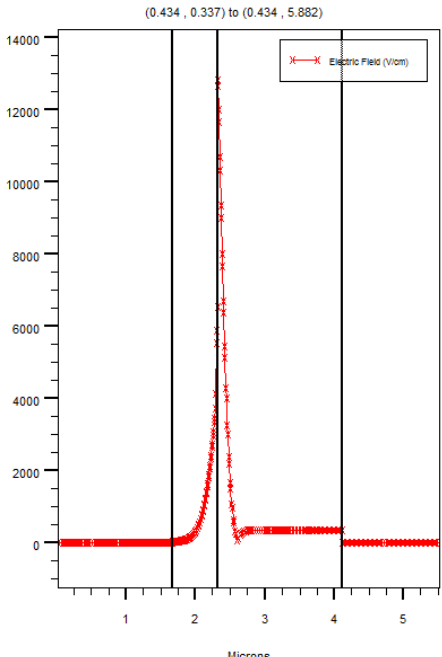

Minons

Fig4. Configuration, current density and electric field of the P3HT/CdSe solar cells with doping $13 \times 10^{14} \mathrm{~cm}^{-3}$ and $16 \times 10^{14} \mathrm{~cm}^{-3}$ in electron donor and hole donor, respectively. 

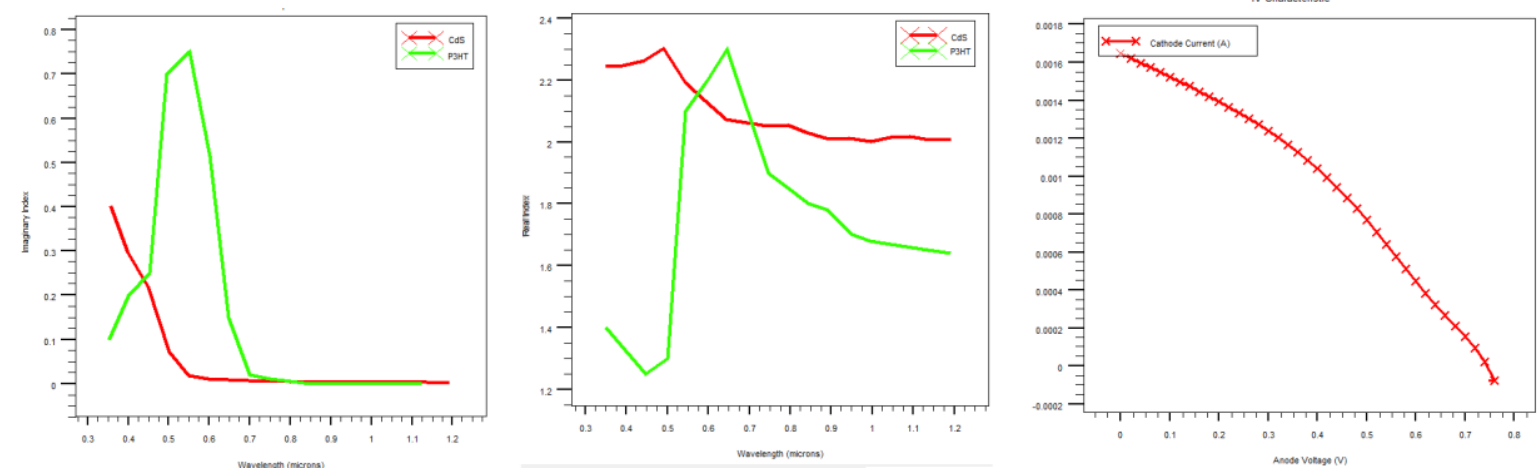

Fig5. Refractive index and current-voltage curve of the P3HT/CdSe solar cells with doping $13 \times 10^{14} \mathrm{~cm}^{-3}$ and $16 \times 10^{14} \mathrm{~cm}^{-3}$ in electron donor and hole donor, respectively.

\section{Regulating Doping In Hole Donor}

We consider $1.6 \times 10^{15} \mathrm{~cm}^{-3}$ for doping in hole donor.
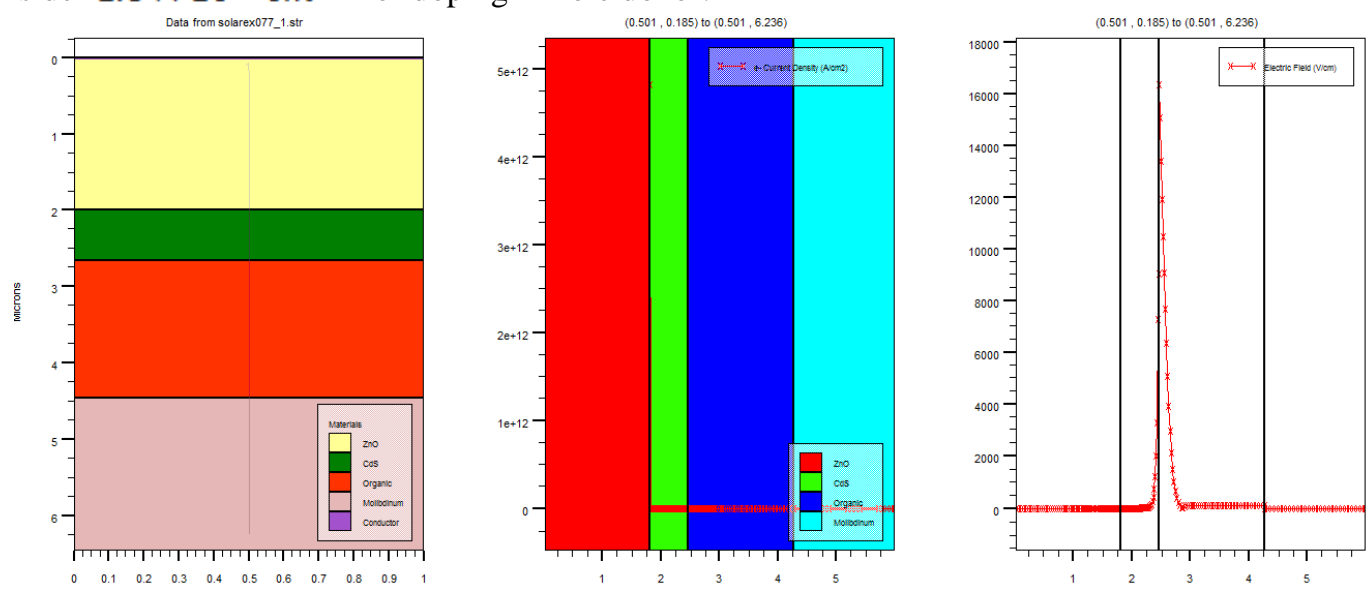

Fig6. Configuration, current density and electric field of the P3HT/CdSe solar cells with doping $1.6 \times 10^{15} \mathrm{~cm}^{-3}$ in hole donor.

After optimization, the concentration value of $1.6 \times 10^{13} \mathrm{~cm}^{-3}$ was found for doping in hole donor.

Eonsentife

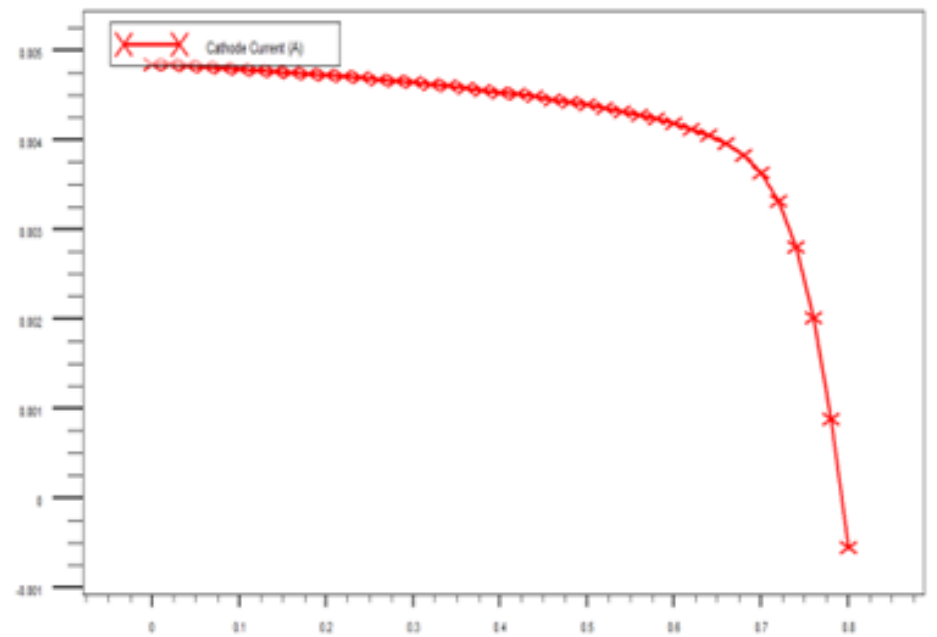

Fig3. Current- voltage curve of the P3HT/CdSe solar cells with doping $1.6 \times 10^{13} \mathrm{~cm}^{-3}$ in hole donor. 
We consider $1.6 \times 10^{15} \mathrm{~cm}^{-3}$ for doping in hole donor.
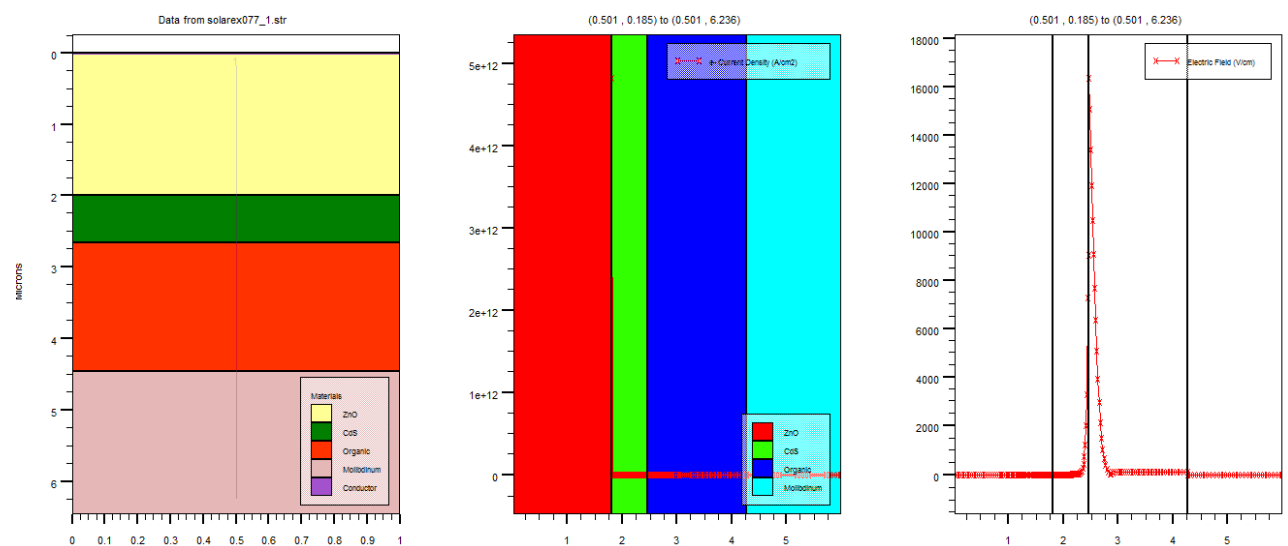

Fig6. Configuration, current density and electric field of the P3HT/CdSe solar cells with doping $1.6 \times 10^{15} \mathrm{~cm}^{-3}$ in hole donor.

After optimization, the concentration value of $1.6 \times 10^{13} \mathrm{~cm}^{-3}$ was found for doping in hole donor.

ronsinte

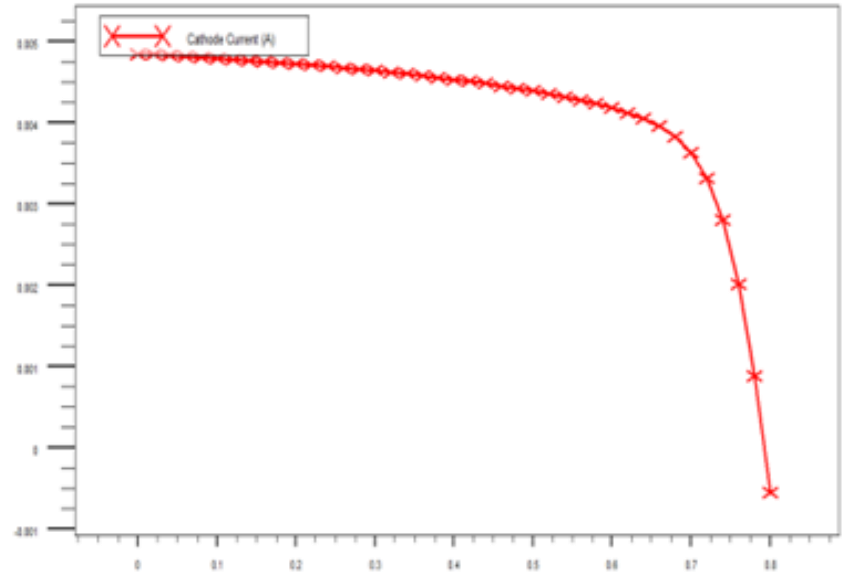

Fig3. Current- voltage curve of the P3HT/CdSe solar cells with doping $1.6 \times 10^{13} \mathrm{~cm}^{-3}$ in hole donor.
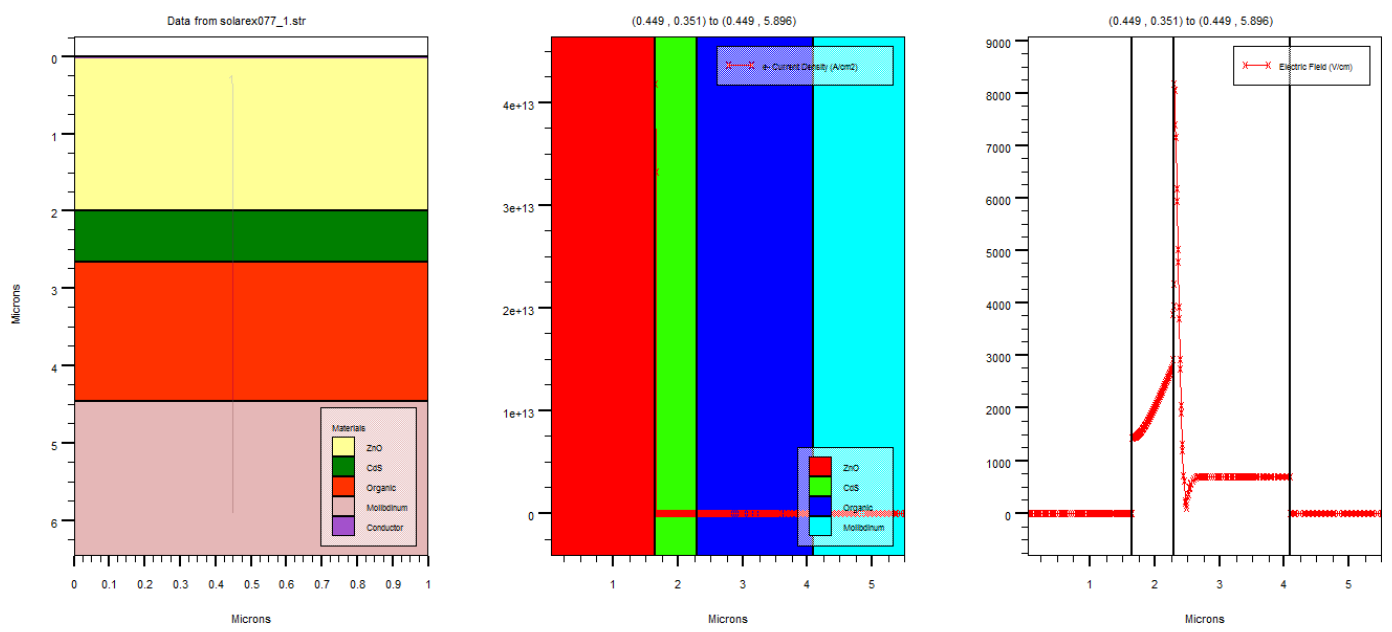

Fig4. Configuration, current density and electric field of the P3HT/CdSe solar cells with doping $1.6 \times 10^{13} \mathrm{~cm}^{-3}$ in hole donor. 
Table 1.show the comparison between the achieved output parameters for our optimized ZnO/P3HT : CdSe /Mo with the achieve results in [15] and [16], which indicates good improvement in both PCE and FF.

\begin{tabular}{|c|c|c|c|}
\hline Cell & Efficiency (PCE) & Fill Factor (FF) & Ref. \\
\hline Zno/P3Ht:CdSe/Molybdenum & 1.248 & 0.78 & This work \\
\hline ITO/P3HT:CdSe/Ag & 0.14 & 0.3 & {$[15]$} \\
\hline ITO/ZNO/PCBM/MOO3 & 0.31 & 0.42 & {$[16]$} \\
\hline
\end{tabular}

\section{Conclusion}

In this article, first, we analyzed the best doping for the configuration of $\mathrm{P} 3 \mathrm{HT} / \mathrm{CdSe}$ in order to improve the performance of the solar cell. For this aim, we investigated the current density of electrons, the electric field, the short-circuit current, and the open-circuit voltage in different doping . The results indicate that when the doping is increased in $\mathrm{P} 3 \mathrm{Ht}$ and is decreased in CdSe, the current density of electrons, the electric field, the short-circuit current, and the open-circuit voltage are increased. Finally, we obtained doping of $13 \times 10^{14} \mathrm{~cm}^{-3}$ and $16 \times 10^{14} \mathrm{~cm}^{-3}$ for electron donor and hole donor as the optimum doping for this configuration.

\section{References}

[1]. H. Altas. 2007. A Photovoltaic Array Simulation Model for Matlab-Simulink GUI Environment. Dept. of Electrical and Electronics Engineering, Karadeniz Technical University, Trabzon, Turkey. IEEE.

[2]. J.M. Enrique.2007. Theoretical assessment of the maximum power point tracking efficiency of photovoltaic facilities with different converter topologies. Departamento de Ingenierı'a Electro' nica, de Sistemas Informa' ticos y Automa' tica, Universidad de Huelva, Spain

[3]. Ismail H. Altas.2007. A Novel Photovoltaic Online Search Algorithm for Maximum Energy Utilization. Department of Electrical and Electronics Engineering 61080, Trabzon - Turkey

[4]. Marcelo Gradella Villalva. MAY 2009. Comprehensive Approach to Modeling and Simulation of Photovoltaic Arrays. IEEE Transactions on Power Electronics, Vol. 24, No. 5

[5]. Dezso Sera, Student Member. July 2008. Optimized Maximum Power Point Tracker for Fast-Changing Environmental Conditions. IEEE Transactions on Industrial Electronics, Vol. 55, No. 7

[6]. Kashif Ishaque a, Zainal Salam.2011. Modeling and simulation of photovoltaic (PV) system during partial shading based on a twodiode model. Faculty of Electrical Engineering, Universiti Teknologi Malaysia, UTM 81310, Skudai, Johor Bahru, Malaysia Kumamoto University, 2-39-1 Kurokami, Kumamoto 860-8555, Japan

[7]. Nasr Eddine Chabane Sari, Prof.2014. Electrical stimulation of organic solar cell based on CuPc/C60 heterojunctions. IEEE

[8]. Trung Dang Minh. 12 November 2015.P3HT:PCBM, best seller in polymer photovoltaic research. DOI: 10.1002/adma.201100792 Source: PubMed

[9]. Zhengguo Xiao, Qingfeng Dong.2015. Efficiency Enhancement in Polymer Solar Cells With a Polar Small Molecule Both at Interface and in the Bulk Heterojunction Layer. IEEE JOURNAL OF PHOTOVOLTAICS, VOL. 5, NO. 5.

[10]. Matthew J. Greaney and Richard L. Brutchey.2015. Ligand engineering in hybrid polymer: nanocrystal solar cells. Materials Today, Volume 18, Number 1.

[11]. Shenqiang Ren, Liang-Yi Chang, 2011. Inorganic_Organic Hybrid Solar Cell: Bridging Quantum Dots to Conjugated Polymer Nanowires.nano letter.

[12]. Patrick R. Brown.2014. Energy Level Modification in Lead Sulfide Quantum Dot Thin Films through Ligand Exchange. American Chemical Society

[13]. Liang Shen, Wenjuan Yu.2013. Performance Improvement of Low-Band-Gap Polymer Solar Cells by Optical Microcavity Effect. IEEE ELECTRON DEVICE LETTERS, VOL. 34, NO. 1.

[14]. Ehsan H. Sabbar, Mustafa H.2012. A Fabricated Solar Cell from ZnO/a-Si/Polymers.

[15]. International Journal of Advanced Science and Technology Vol. 44, July.

[16]. A. Sánchez-Martínez and Etal,2015. Solution Processable P3HT/CdS Hybrid Solar Cells with Different Layer Configurations. Int. J. Electrochem. Sci., Vol. 10

[17]. Wenbo Wang and Etal,2014. Optical simulations of P3HT/Si nanowire array hybrid solar cells.Nanoscale Research Letter 